\title{
INVESTIGATIONS ON WATER CIRCULATION IN ANIMAL SEA-WATER BASINS - ON THE EXAMPLE OF SEALS' BREEDING POOLS
}

Piotr Zima

Gdansk University of Technology, Poland

\begin{abstract}
This paper presents general comments concerning investigations on water circulation in animal breeding pools containing sea water. As an example are given results of computer simulation of water circulation in seals' breeding pools situated in Marine Station at Hel, belonging to Oceanographic Institute, Gdansk University. A mathematical model of three main pools was prepared with taking into account their inflow and outflow water supply points. Next, the object indication ( tracer) tests were done with the use of mathematical modelling as well as in-situ measurements. For description of flow field in steady conditions a simplified model of 2D flow in the form of Helmholtz biharmonic equation of stream function, recalculated then into velocity vector components, was used. The equation, supplemented with appropriate boundary conditions, was solved numerically by using the finite differences method. The spreading of a substance dissolved in water (tracer) was analyzed by solving 2D equation of transient advecting - dispersing transport. To solve it the finite volumes method was applied. The applied model was verified by conducting the indication tests with the use of the rhodamine WT as a tracer. The obtained results made it possible to reconstruct water circulation within the seals' pools and identify stagnation zones in which water circulation may be made difficult.
\end{abstract}

Keywords: circulation of water in animal breeding pool, water flow through the pools, computer simulation,

\section{INTRODUCTION}

Circulation of water in animal breeding pools is an important factor which influences its quality. It is especially important in case of basins where warm-blooded animals live. The animals live in a limited space and influence parameters of water in the pools during feeding and by excreting metabolism products which contaminate the water. To prevent it, pools of the kind are fitted with water exchange systems. Their main task is to ensure an appropriate water change rate. They should also ensure an appropriate circulation to prevent forming the stagnation zones which may lead to growing concentration of contamination or microbes. (Lehnert et al., 2014). The detrimental phenomena can be counteracted by extending the zone of active exchange of water as well as by forming appropriate hydraulic conditions aimed at elimination of stagnation zones. This can be done by a few ways. One of them is the mathematical modelling of flow field (Zima, 2014) as well as the conducting of tracer tests (numerical simulations and indication tests in situ). Unfortunately, mathematical modelling has one important drawback consisting in that to solve converse problems is not possible (when demands are imposed on problem solution and considered system geometry has to be solved, and/or limit conditions which make it possible to satisfy the demands). It allows only to analyze the accepted solutions and try to find a solution which satisfies the assumed criterion the best.

As far as the sea-water pools are concerned, additional problems should be expected. Circulation of water in such objects consists in delivering sea water during its exchange. It may cause problems dealing with moving contaminations from open water area and deposing them within closed ecosystem of animal breeding pools. It concerns all kinds of contaminations (biological, chemical and physical ones). Among physical ones, the more and more often present microplastic forms should be mentioned (Setälä et al, 2014). On the other hand, an aggressive impact of sea water on elements of water exchange systems should be remembered. This is especially dangerous in case when changes of pump 
working parameters occur along with time (Puzyrewski et al. 2010, Szantyr et al. 2011). Therefore even at a water exchange system correctly designed and tested it is necessary to control periodically operation of pump systems, and, at changed parameters of their operation - to analyze their impact on water circulation within pools.

\section{METHODS USED FOR INVESTIGATIONS IN QUESTION}

As already mentioned, various methods may be applied to investigate water circulation in animal breeding pools. Good results can be achieved by means of mathematical modelling with the use of computer simulations. In order to perform mathematical modelling tasks it is necessary to prepare data for such model (a mathematical abstraction) which represents a real object (Meerschaert 2007) and to solve appropriate mathematical physics equations, which describe water flow (Szymkiewicz 1991, 2010, Szewc et al. 2012a), and in case of tracer tests - behaviour of the tracer in the form of dissolved substance in the calculated velocity field (or a twophase medium - Szewc et al. 2012b). The first phase, i.e. the forming of a mathematical model of real object, requires to define a calculation area, i.e. geometry of a pool in question. In case of animal breeding pools filled with sea water, various techniques of performing measurements which allow to use the more and more exact methods, may be implemented, depending on their size. In case of large pools, techniques used in sea-bed bathymetry are applied (Kozaczka et al. 2012, Grelowska et al. 2013, Kozaczka 2013, Grelowska and Kozaczka 2014), for small pools geodesic measurements are satisfactory (Burdziakowski et al., 2015). Apart from area of a pool, it is necessary to know parameters which describe water circulating system, namely: pump capacity and location of water inflows to and outflows from the pool .

The next step is to perform computer simulations. General motion equations of non-homogenous liquid represent an extremely powerful tool at contemporary development level of numerical methods and today capabilities of computers. The tool consists in far -leading formalization exemplified by commercial computer software. Its application becomes formally more and more simple, that results in growing popularity of applying Computational Fluid Dynamics in environment engineering (Lipeme Kouyi et al. 2003, Zima et al., 2009). However, application of such programs for real objects, at satisfying an appropriate accuracy level, requires huge resources associated with preparation of a calculation model (generation of calculation area), and running the simulations, appears often very time-consuming. For this reason, simplified models which allow for utilizing specific features of an object and reaching good quality results, are commonly used. In this paper an example of such approach is presented. To determine water velocity field in seals' breeding pools, 2D kinematic model in the form of Helmholtz biharmonic equation was applied. It allows for reproduction of velocity field in steady- state conditions (that is equivalent to specificity of water circulation in objects of the kind) as well as to draw trajectories of particles indicating stagnation zones. However it does not provide a full picture of changes in concentration of a factor which contaminates water in the pool. For this reason also tracer tests should be performed by simulating introduction of a tracer in the form of a substance dissolved in water in trouble spots of the pool. Migration of the tracer can be revealed by solving the dissolved substance transport equation. In the case in question, 2D equation of unsteady advecting- dispersing transport of dissolved substance was applied (Sawicki and Zima, 1997).

The tracer tests should be conducted by numerical simulating as well as indication tests on the object in situ. To the indication tests, substances harmless for animals living in the pools, should be applied (Zima, 2014). In the presented example the indication tests were conducted with the use of the tracer in the form of the rhodamine WT. Portions of an appropriately dissolved tracer were delivered into characteristic spots, and then measurements of changes in rhodamine concentration along with time were made in selected points of the pools. Two Cyclop 7 sounders of Terner Designs were used for the measurements.

\section{MATHEMATICAL MODEL}

In producing the mathematical model a schematization of real object should be first of all made. In the model an idealization of real conditions should be built in order to accept appropriate assumptions simplifying mathematical description of the phenomenon in question (Meerschaert, 2007). In this paper results of the investigations performed in the seals' breeding pools of Marine Station at Hel, Institute of Oceanography, Gdańsk University, are presented as an example. The arrangement and basic data concerning the pools are shown in Fig. 1 where water inflows and outflows are also depicted. The analysis covered water flow through three main pools (No. 3, 4 and 7), in which seals live. Depth of the pools is different and changes from $h=1.0 \mathrm{~m}$ up to $h=2.9 \mathrm{~m}$.

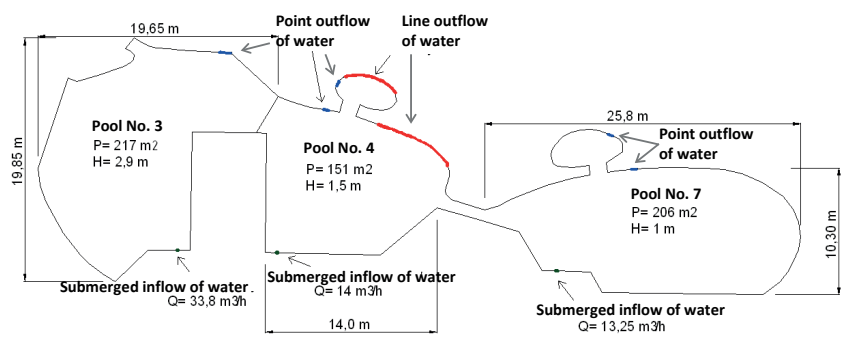

Fig.1. The assumed physical model of the seals' breeding pools

The next stage of building the mathematical model is to accept a mathematical description of run of the phenomenon . First, the description model of steady flow field at different water depth was assumed (the particular pools have different depth values and are connected to each other). As already mentioned above, was taken the kinematic model in which, 
apart from the satisfying of mass conservation equation, also kinematic condition concerning fluid element rotation is fulfilled (Anderson, 1995). The model assuming changing rotation of fluid element is commonly in use. In case of 2D vector velocity: $\mathbf{u}=\left[u_{x}, u_{y}\right]$ the condition can be written as follows:

$$
\operatorname{rot} \mathbf{u}=\Omega(x, y)
$$

where: $\Omega(x, y)$ - the assumed rotation distribution function.

If the Stokes assumption is accepted (on neglecting inertia forces) for steady flow conditions and constant temperature of water, with taking into account Eq. (1), the following is obtained:

$$
\Delta \Omega=0
$$

It is the equation for the harmonic function $\Omega$, called Helmholtz equation. If the stream function $\psi(x, y)$ is defined as follows:

$$
\frac{\partial \psi}{\partial y}=h \cdot u_{x}, \quad-\frac{\partial \psi}{\partial x}=h \cdot u_{y}
$$

(where $h$ stands for water depth), Eq. (2) (biharmonic one) can be expressed in the following form:

$$
\Delta \Omega=\Delta \Delta \psi=\Delta\left(\frac{1}{h} \Delta \psi-\frac{1}{h^{2}} \nabla \psi \nabla h\right)=0
$$

Eq. (4) may be interpreted to be a generalization of kinematic models in which velocity rotation is a harmonic function described by Laplace equation. In the general case Dirichlet problem should be solved for biharmonic operator. Solution of the problem in 2D space $(x, y)$ is the stream function $\psi(x, y)$. With the use of Eq. (3) velocity vector components can be calculated over the whole area .

Next, was assumed the migration model for dissolved substance (tracer), which, in unsteady conditions, at changeable depth as well as $2 \mathrm{D}$ space, may be written in the following form (Sawicki and Zima, 1997):

$$
\begin{aligned}
& \frac{\partial h c}{\partial t}+\frac{\partial\left(h u_{x} c\right)}{\partial x}+\frac{\partial\left(h u_{y} c\right)}{\partial y}=\frac{1}{h} \frac{\partial}{\partial x}\left(h D_{x x} \frac{\partial c}{\partial x}+h D_{x y} \frac{\partial c}{\partial y}\right) \\
& +\frac{1}{h} \frac{\partial}{\partial y}\left(h D_{y x} \frac{\partial c}{\partial x}+h D_{y y} \frac{\partial c}{\partial y}\right)
\end{aligned}
$$

where: $t$ - time, $c$ - concentration of dissolved substance; and, coordinates of the dispersion tensor $\mathbf{D}$ may be defined as follows:

$$
D_{\mathrm{Ix}}=D_{L} n_{x}^{2}+D_{T} n_{y}^{2}, D_{y}=D_{y}=\left(D_{L}-D_{T}\right) n_{x} n_{y}, D_{y}=D_{L} n_{y}^{2}+D_{T} n_{x}^{2}
$$

where: $n=\left[n_{x}, n_{y}\right]$ is the velocity directional vector:

$$
n_{x}=\frac{u_{x}}{|\mathbf{u}|}, n_{y}=\frac{u_{y}}{|\mathbf{u}|}
$$

The longitudinal, $D_{L}$, and transverse, $D_{T}$, coordinates of the dispersion tensor (6) are described by the following formula (Elder, 1956):

$$
\begin{array}{ll}
D_{L}=\alpha \cdot h \cdot v^{*} & 30<\alpha<3000 \\
D_{T}=\beta \cdot h \cdot v^{*} & 0.15<\beta<0.30
\end{array}
$$

where: $v^{*}$-dynamical velocity. In this paper values of the coefficients $\alpha$ and $\beta$ are determined on the basis of available literature sources. Finally, $\alpha=300$ and $\beta=0.23$ was assumed. Eq. (5) constitutes mathematically an initial - boundary value problem, which, to be solved, requires assuming initial condition and boundary ones.

Eq. (4) and (5), in general cases, can be solved only by using numerical methods. In this work the finite difference method was used for biharmonic equation (Anderson, 1995) and the finite volume method - for dissolved substance transport (LeVeque, 2002). To apply the above mentioned methods the assumed solution area was split into square cells of $\Delta x=\Delta y=0.25 \mathrm{~m}$ (Fig.2). For solving the problem the authors' original programs were used.

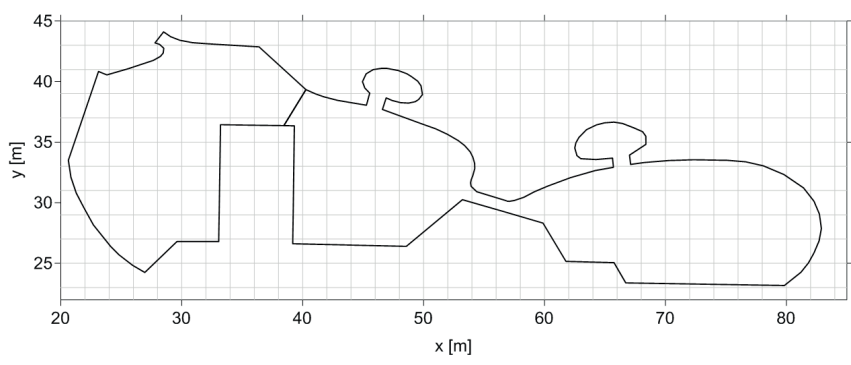

Fig.2. The assumed calculation area

\section{COMPUTER SIMULATIONS OF WATER FLOW THROUGH THE POOLS}

To obtain a solution of the biharmonic equation (4) it is necessary to take into account water supply to the seals' breeding pools. In Fig. 1 location of water inflow and outflow spots in particular pools, is indicated. In these spots, in the mathematical model, the boundary condition simulating water inflow and outflow was assumed. Next, computer simulations were carried out in steady motion conditions. 


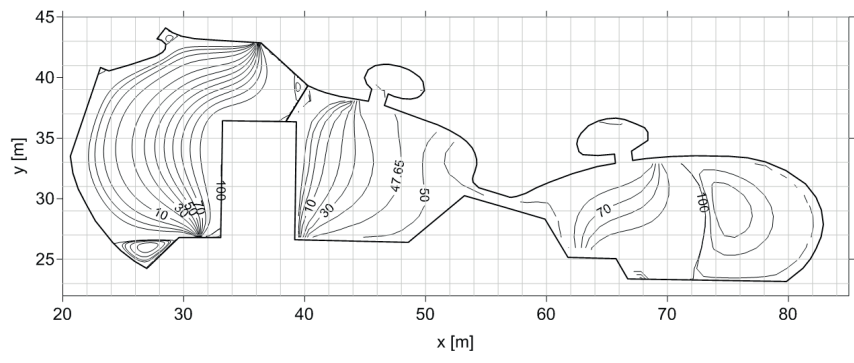

Fig. 3. Distribution of the stream function $\psi(x, y)$ in steady motion conditions in the seals' breeding pools

The obtained results in the form of distribution of the stream function $\psi(x, y)$ are presented in Fig. 3 (from 0 up to $100 \% \mathrm{~m}^{3} / \mathrm{s}$ per unit of depth). The zones inside closed lines of the stream function indicate areas of closed circulation of water. Then, by using Eq. (3), velocity distribution was calculated over the entire defined area. Results of the calculation are presented in Fig. 4. Maximum velocity values (in the spots of water flow into the pools) were equal to $0.5 \mathrm{~m} / \mathrm{s}$ (the longest vectors shown in Fig. 4).

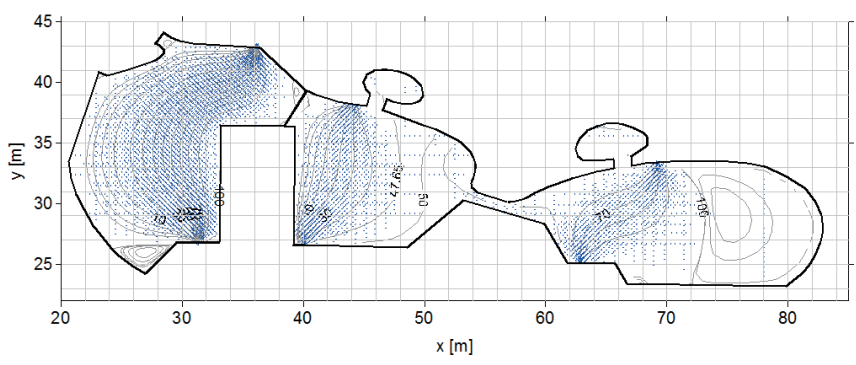

Fig. 4. Distribution of the water velocity field in steady motion conditions in the seals' breeding pools

Next, simulations of flow of the tracer introduced to inflow spots of each of the seals' breeding pools, were conducted under assumption on continuous delivery of the tracer $(100 \%$ amount). Their results are given in Fig. 5. The performed calculations made it possible to estimate duration time of flow of the tracer up to outflow spot. For all the pools the time was equal to about $6 \div 8 \mathrm{~min}$.

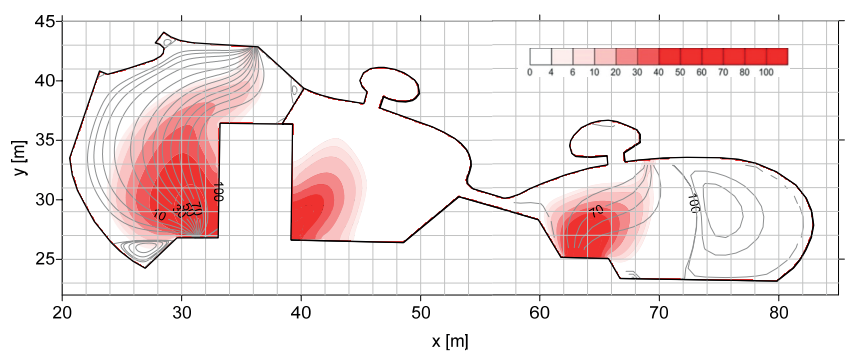

Fig. 5. Distribution of tracer concentration after reaching the spots of water outflow from the seals' breeding pools
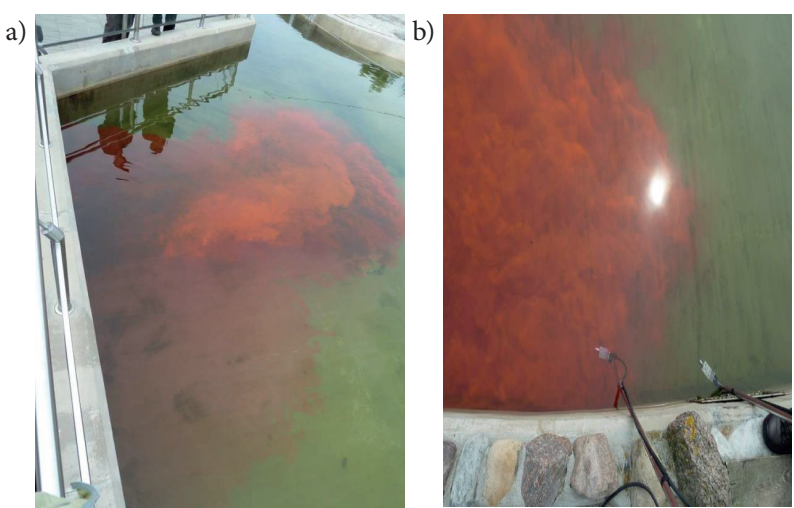

Fig. 6. Tracer tests in the pool No. 7 :

a) input of dosage of the rhodamine WT at the spot of water inflow to the pool, b) continuous measurement of concentration of the rhodamine WT at the spot of water outflow from the pool

In order to verify the achieved results, the tracer tests were conducted in situ in the seals' breeding pools. $20 \%$ rhodamine WT was applied as a tracer. $100 \mathrm{ml}$ dosage was introduced to the spot of water inflow to each of the pools, and in the outflow spots continuous measurements were conducted by means of two Cyclop 7 sounders (Fig.6 - tracer tests in the pool No. 7). The tests were carried out in each of the pools separately (during the tests no seals were inside the pools). The example results, in the form of changes of concentration along with time, observed in the spot of outflow from the pool No. 3, are presented in Fig. 7 (where continuous line stands for measurement, broken line - result of numerical simulations).

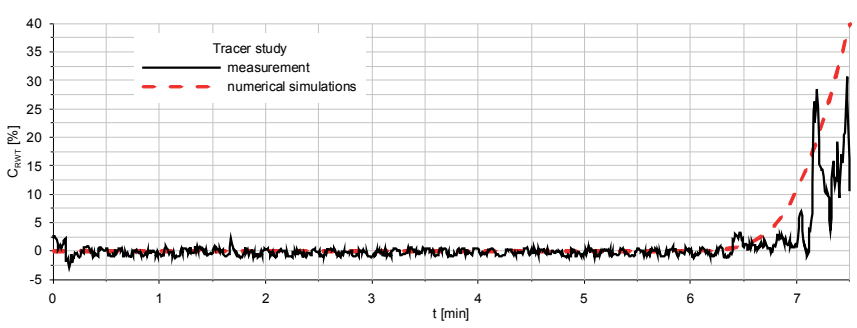

Fig. 7. Measurement results of concentration of the rhodamine WT at outflow from the pool No. 3, and results of numerical simulations - measurement of time of reaching, by the tracer, the outflow spot from the pool No. 3

\section{DISCUSSION OF THE OBTAINED RESULTS AND CONCLUSIONS}

The performed computer calculations made it possible to draw the stream function field $\psi(x, y)$ and obtain values of the components $u_{x}(x, y)$ and $u_{y}(x, y)$ of the velocity field $\mathbf{u}$ in the tested seals' breeding pools. The distribution and shape of isolines of the stream function showed the arrangement of main directions of water circulation as well as location of outflow-free (stagnation) zones within the pools. In the pool No. 3 such zones occur mainly in its corners, and the largest one is situated just behind the inflow delivering water to the pool. In the pool No. 4 water flows in the form of uniform streams from inflow to outflow and builds the stagnation 
zone in the channel connecting the pools No. 4 and 7. In the pool No. 7 the main stream of water circulation is formed along the streams connecting inflow and outflow spots. In the pool was built a vast stagnation zone in which exchange of water is made difficult and which does not take part in circulation of water flowing through the pool. The highest velocities occur at inflows and outflows (between 0.48 and $0.51 \mathrm{~m} / \mathrm{s}$ ), and next, inside the pool, they drop to the value of a few centimeters per second only. All the stagnation zones are characteristic of closed water circulation in which velocity values are on the level below one centimeter per second. Next, the mathematical modelling of spreading the tracer was performed for each of the pools. The problem consisting in simulation of introduction of the tracer into inflow spots to each of the pools was solved and its spreading was calculated. The computer simulations were verified by means of the tests in situ. The conducted tracer tests showed that duration time of arriving the outflow by the tracer particles was between 6 and $8 \mathrm{~min}$ for all the pools. The calculation results confirmed the conducted measurements. Therefore it seems that application of the proposed approach in the stage of formulating the design guidelines for the animal breeding objects of the type is justified, hence it is also suggested to use the gained experience in case of modernization of other objects intended for the breeding of water animals so as to obtain such form of water exchange in an object, which could ensure an appropriate change rate of the whole water inside the pool (of at least a few times per day)

\section{BIBLIOGRAPHY}

1. Anderson J. D.: Computational Fluid Dynamics. The Basics with Applications, McGraw-Hill Inc., New York, USA (1995), pp. $1 \div 547$

2. Burdziakowski P., Janowski A., Kholodkov A., Matysik K., Matysik M., Przyborski M., Szulwic J., Tysiąc P., Wojtowicz A.: Maritime Laser Scanning As The Source For Spatial Data, Polish Maritime Research, Vol. 22, No. 4(88) (2015), pp. $9 \div 14$

3. Elder J.W.: The dispersion of marked fluid in turbulent shear flow, J. of Fluid Mech., Vol. 5, No. 4 (1956), pp. $544 \div 560$

4. Grelowska G., Kozaczka E., Kozaczka S.: Gdansk Bay sea bed sounding and classification of its results, Polish Maritime Research, Vol. 20, No 3 (2013), pp. 45 $\div 50$

5. Grelowska G., Kozaczka E.: Underwater Acoustic Imaging of the Sea, Archives of Acoustics, Vol. 39, No. 4 (2014), pp. $439 \div 452$

6. Kozaczka E., Grelowska G., Kozaczka S., Szymczak W.: Processing data on sea bottom structure obtained by means of the parametric sounding, Polish Maritime Research, Vol. 19, No 4(76) (2012), pp. $3 \div 10$
7. Kozaczka E.: Acoustics waves in the sea, Archives of Acoustics, Vol. 38, No. 3 (2013), pp. $441 \div 442$

8. Lehnert K., Müller S., Weirup L., Ronnenberg K., Pawliczka I., Rosenberger T., Siebert U.: Molecular Biomarkers in Grey Seals (Halichoerus grypus) to Evaluate Pollutant Exposure, Health and Immune Status, Marine Pollution Bulletin, Vol. 88, No. $1 \div 2$ (2014), pp. $311 \div 318$.

9. LeVeque R. J.: Finite Volume Method for Hyperbolic Problems, Cambridge University Press, New York, USA (2002), pp. $1 \div 558$

10. Lipeme Kouyi, G.,Vazquez, J., Poulet, J.: 3D free surface measurement and numerical modelling of flows in storm overflows, Flow Measurement and Instrumentation, Vol.14, No. 3 (2003), pp. $79 \div 87$

11. Meerschaert M. M.: Mathematical Modeling, 4 ed. Elservier Science (2013), pp. 365

12. Puzyrewski R., Żochowski K., Flaszyński P.: A Method for Analyzing Ram Pressure Characteristics of Impeller Pump Rotor, Polish Maritime Research, Vol. 17, No. 2(64) (2010), pp. $52 \div 57$

13. Sawicki J.M; Zima P.: The Influence of Mixed Derivatives on The Mathematical Simulation of Pollutants Transfer, $4^{\text {th }}$ International Conference on Water Pollution, Bled, Slovenia (1997), pp. $627 \div 635$

14. Setälä O., Fleming-Lehtinen V., Lehtiniemi M.: Ingestion and Transfer of Microplastics in The Planktonic Food Web, Environmental Pollution, Vol. 185 (2014), pp. $77 \div 83$

15. Szantyr J., Flaszyński P., Tesch K., Suchecki W., Alabrudziński S.: An Experimental and Numerical Study of Tip Vortex Cavitation, Polish Maritime Research, Vol. 18 , No. 4 (2011), pp. $14 \div 22$

16. Szewc K., Pozorski J., Minier J.: Analysis of The Incompressibility Constraint in The Smoothed Particle Hydrodynamics Method, International Journal for Numerical Methods in Engineering, Vol. 92, No. 4 (2012a), pp. $343 \div 369$

17. Szewc K., Taniere A., Pozorski J., Minier J.: A Study on Application of Smoothed Particle Hydrodynamics to MultiPhase Flows, International Journal of Nonlinear Sciences and Numerical Simulation (2012b), pp. $383 \div 395$

18. Szymkiewicz R.: Numerical Modeling in Open Channel Hydraulics, Book Series: Water Science and Technology Library, vol. 83 (2010), pp. 1-419;

19. Szymkiewicz R.: Finite-Element Method for the Solution of the Saint-Venant Equations in an Open Channel 


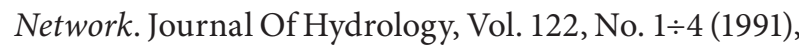
pp. $275 \div 287$

20. Zima P.: Numerical Simulations and Tracer Studies as a Tool to Support Water Circulation Modeling in Breeding Reservoirs, Archives of Hydro-Engineering and Environ-

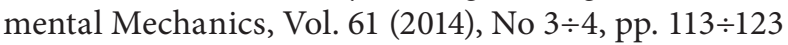

21. Zima, P., Mąkinia, J., Swinarski, M., Czerwionka, K.: Combining Computational Fluid Dynamics with a Biokinetic Model for Predicting Ammonia and Phosphate Behavior in Aeration Tanks, Water Environment Research, Vol. 81, No. 11 (2009), pp. 2353 $\div 2362$.

\section{CONTACT WITH THE AUTHOR}

\section{Piotr Zima}

Civil Engineering and Environment Faculty Gdańsk University of Technology

11/12 Narutowicza St. 80 - 233 Gdańsk

Poland 